\title{
APRENDIZAJE BASADO EN PROYECTOS Y SU EVALUACIÓN \\ FORMATIVA EN LA ASIGNATURA FUNDAMENTOS Y ESTRATEGIAS \\ DIDÁCTICAS DE LA EDUCACIÓN MUSICAL. UNA BUENA PRÁCTICA EN \\ LAUNIVERSIDAD DE VALLADOLID
}

"Project-Based Learning and its Formative Evaluation in the subject Fundamentals and Didactic Strategies of Music Education. A good practice in the University of Valladolid

Aprendizagem baseada em projetos e sua avaliação formativa na disciplina Fundamentos e estratégias didáticas da educação musical. Uma boa prática na Universidade de Valladolid

Inés María Monreal Guerrero (1) María de la O Cortón de las Heras (2)

David Carabias Galindo (3)

(1) Universidad de Valladolid, España. Teléfono: +34921112295. Correo electrónico: ines.monreal@mpc.uva.es

(2) Universidad de Valladolid, España. Teléfono: +34921112295. Correo electrónico: mariacorton@mpc.uva.es

(3) Universidad de Valladolid, España. Teléfono: +34921112295. Correo electrónico: carabias@mpc.uva.es

\section{Resumen}

Se presenta una buena práctica educativa universitaria de Aprendizaje Basado en Proyectos y Evaluación Formativa que fue llevada a cabo durante el curso 2018-2019 dentro de la asignatura "Fundamentos y estrategias didácticas de la Educación Musical", que es impartida a los estudiantes de segundo curso del Grado en Educación Primaria de la Universidad de Valladolid. La experiencia que presentamos fusiona la formación inicial innovadora del estudiante con la evaluación formativa de su proceso de aprendizaje. En esta experiencia han participado 44 estudiantes, las valoraciones relacionadas con la experiencia han sido bastante satisfactorias y han posibilitado un aprendizaje procesual enraizado en el nuevo paradigma educativo vinculado a las aulas del s. XXI.

Palabras clave: Evaluación formativa; aprendizaje basado en proyectos; grado educación primaria

Aprendizaje basado en proyectos y su evaluación formativa en la asignatura Fundamentos y Estrategias

Didácticas de la Educación Musical. Una buena práctica en la Universidad de 


\begin{abstract}
A good university educational practice of Project-Based Learning and Formative Assessment is presented, which was carried out during the 2018-2019 academic year within the subject "Fundamentals and Didactic Strategies of Music Education", which is taught to the second-year students of the Degree in Primary Education of the University of Valladolid. The experience that we present fuses the innovative initial training of the student with the formative assessment of their learning process. In this experience 44 students, evaluations related to the experience were quite satisfactory and enabled a processual learning rooted in the new educational paradigm linked to the classrooms of the XXI century.
\end{abstract}

Keywords: Formative assessment; project-based learning; primary education degree

\title{
Resumo
}

É apresentada uma boa prática educativa universitária de Aprendizagem Baseada em Projetos e Avaliação Formativa, realizada durante o ano letivo 2018-2019 na disciplina "Fundamentos e estratégias didáticas de Educação Musical", ensinado a alunos do segundo ano da Liccenciaturaem Educação Primária da Universidade de Valladolid. A experiência que apresentamos interliga a formação inicial inovadora do aluno com a avaliação formativa do seu processo de aprendizagem. Nesta experiência participaram 44 alunos. As avaliações relacionadas com a experiência foram bastante satisfatórias e possibilitaram uma aprendizagem processual enraizada no novo paradigma educativo e vinculado às aulas do século XXI.

Palavras-chave: Avaliação formativa; aprendizagem baseada em projetos; Licenciatura em educação primária

\section{Introducción}

La sociedad de la información y el conocimiento en la que estamos inmersos nos hace plantearnos retos educativos a afrontar (Monreal \& Sobrino, 2014), entre los mismos, y enmarcados dentro de la legislación educativa nacional (ORDEN ECD/65/2015), encontramos la necesidad de generar planes de acción con el que se busque conseguir un resultado práctico al aprendizaje autónomo que se está desarrollando dentro del aula.

Como aparecía en la citada Orden:

Aprendizaje basado en proyectos y su evaluación formativa en la asignatura Fundamentos y Estrategias

Didácticas de la Educación Musical. Una buena práctica en la Universidad de Valladolid 
El trabajo por proyectos (...) pretende ayudar al alumnado a organizar su pensamiento favoreciendo en ellos la reflexión, la crítica, la elaboración de hipótesis y la tarea investigadora a través de un proceso en el que cada uno asume la responsabilidad de su aprendizaje, aplicando sus conocimientos y habilidades a proyectos reales. (p. 18)

Por ello consideramos necesario sentar las bases formativas para que adquieran herramientas conducentes al diseño y evaluación de proyectos que, en un futuro, puedan ser implementados en su fase de prácticum o en un aula de primaria.

El Aprendizaje Basado en Proyectos (ABP) es un conjunto de experiencias de aprendizaje que involucran a los estudiantes en proyectos complejos del mundo real, a través de los cuales desarrollan y aplican habilidades y conocimientos (Zavala, 2007).

Además, favorece la adquisición de distintas competencias basadas en la experimentación, comunicación e investigación (Galeana, 2006).

El ABP proporciona una experiencia de aprendizaje activo en el estudiante le involucra en un proyecto complejo y significativo, mediante el cual desarrolla integralmente sus capacidades, habilidades, actitudes y valores (Estrada, 2012). Se acerca a una realidad concreta en un ambiente académico, por medio de la realización de un proyecto de trabajo (Martí, Heydrich, Rojas \& Hernández, 2010). Estimula en los estudiantes el desarrollo de habilidades para resolver situaciones reales, con lo cual se motivan a aprender; los estudiantes se entusiasman con la investigación, la discusión y proponen y comprueban sus hipótesis, poniendo en práctica sus habilidades en una situación real (Pérez, 2008). Esto, unido a la evaluación formativa, entendida como "todo proceso de constatación, valoración y toma de decisiones cuya finalidad es optimizar el proceso de enseñanza aprendizaje que tiene lugar, desde una perspectiva humanizadora y no como mero fin calificador" (Pérez, Julián \& López, 2009, p.35) nos lleva a vincular dicha metodología activa con una evaluación más centrada en el proceso de aprendizaje que constituye una experiencia de aprendizaje en sí misma y que facilita la motivación e implicación del alumnado, así como la mejora de los procesos de enseñanza-aprendizaje en el ámbito universitario (López, 2012).

\section{Contextualización}

En la experiencia que presentamos han participado un total de 44 estudiantes. Se ha desarrollado durante el primer trimestre del curso académico 2018-19 en el Grado de Educación Primaria. La asignatura en la que se ha llevado a cabo es "Fundamentos y estrategias didácticas de la Educación Musical" que tiene una carga de 6 créditos ECTS.

Aprendizaje basado en proyectos y su evaluación formativa en la asignatura Fundamentos y Estrategias

Didácticas de la Educación Musical. Una buena práctica en la Universidad de Valladolid 


\section{Diseño y desarrollo}

Al inicio de la asignatura se presentan los criterios de calificación preestablecidos para con la asignatura y los distintos bloques, los cuales son consensuados entre alumnado y profesorado.

El bloque III de la asignatura se basa en contenidos relativos al ABP, la carga de trabajo del bloque en créditos es de 3 ECTS. Se ha desarrollado la práctica haciendo hincapié en la importancia de trabajar por competencias clave a través de las metodologías activas, en concreto, el Aprendizaje Basado en Proyectos. En el ámbito de la evaluación, se han utilizado fichas de autoevaluación (que nos han orientado sobre la percepción del alumno sobre su aprendizaje, aun cuando no formaban parte de la calificación final) al considerarse dicha herramienta de evaluación íntimamente vinculada con la competencia aprender a aprender. Con respecto a la utilización de herramientas para abordar la coevaluación entre iguales, hemos utilizado fichas de coevaluación que cumplimentaban cuando cada equipo cuando exponía su diseño de ABP el resto de compañeros.

Con respecto a la valoración de la experiencia por parte de los estudiantes, se ha utilizado un cuestionario validado por integrantes de la Red Nacional de Evaluación Formativa y Compartida (Castejón, Santos \& Palacios, 2015), tras recoger los datos, el resultado es el siguiente (Tabla 1).

Tabla 1 .

Valoración de la experiencia

\begin{tabular}{|c|c|}
\hline Ítems del cuestionario & $\begin{array}{l}\text { MEDIA } \\
(1-5)\end{array}$ \\
\hline $\begin{array}{l}\text { 1-¿Se ha negociado la utilización de esta experiencia en la asignatura al } \\
\text { comienzo del curso? }\end{array}$ & 3,11 \\
\hline $\begin{array}{l}\text { 2-¿Crees que esta experiencia te ha ayudado a adquirir competencias } \\
\text { profesionales? }\end{array}$ & 4,32 \\
\hline $\begin{array}{l}\text { 3-¿La evaluación que se ha planteado favorece la adquisición de las } \\
\text { competencias profesionales }\end{array}$ & 4,32 \\
\hline $4-¿$ Consideras útil lo aprendido con esta experiencia? & 4,27 \\
\hline \multicolumn{2}{|l|}{ 5-¿Qué es lo más útil que has aprendido? Que es una experiencia:... } \\
\hline 5.1.-innovadora, porque desarrolla soluciones nuevas o creativas & 4,09 \\
\hline 5.2.-efectiva, porque demuestra un impacto positivo y tangible de mejora & 4,03 \\
\hline $\begin{array}{l}\text { 5.3.-sostenible, porque se mantiene en el tiempo y puede producir efectos } \\
\text { duraderos }\end{array}$ & 3,93 \\
\hline $\begin{array}{l}\text { 5.4.-replicable, cuando es posible utilizarla como modelo para desarrollarla en } \\
\text { otros contextos }\end{array}$ & 4,25 \\
\hline 6-Señala la satisfacción global en relación con la experiencia & 4,09 \\
\hline 7-Señala la satisfacción global en relación con la evaluación de la experiencia & 4,03 \\
\hline 8-¿Cuál es el grado de dificultad de la experiencia? & 3,43 \\
\hline
\end{tabular}

Aprendizaje basado en proyectos y su evaluación formativa en la asignatura Fundamentos y Estrategias Didácticas de la Educación Musical. Una buena práctica en la Universidad de Valladolid 


\section{Evaluación}

La buena práctica o experiencia educativa se ha evaluado a través del análisis de los datos obtenidos en el cuestionario, parte de dichos resultados aparecen en la Tabla 1 del presente texto. No sólo se ha utilizado el cuestionario, también las exposiciones orales de los diseños de los ABP elaborados por los distintos equipos y el proceso de desarrollo de estos. Con la autoevaluación se ha abordado la autopercepción de sus propias limitaciones al abordar una experiencia innovadora para ellos.

\section{Conclusiones}

A tenor de los datos que arroja la evaluación de la experiencia, afirmamos que los estudiantes han considerado útil lo aprendido, les ha parecido una experiencia innovadora, efectiva, sostenible y un modelo que se puede desarrollar en otros contextos. El grado de satisfacción global en relación con la evaluación de la experiencia también ha sido alto.

\section{Referencias}

Castejón, F.J., Santos, M.L., \& Palacios, A. (2015). Cuestionario sobre metodología y evaluación en formación inicial en educación física / Questionnaire on methodology and assessment in physical education initial training. Revista Internacional de Medicina y Ciencias de la Actividad Física y el Deporte, 15 (58), 245-267.

Recuperado

de: http://cdeporte.rediris.es/revista/revista58/artescala566.htm

Galeana de la O, L. (2006). Aprendizaje basado en proyectos. Investigación en Educación a Distancia, 1, 1-17. Recuperado de: http://ceupromed.ucol.mx/revista/PdfArt/1/27.pdf

Estrada, A. (2012). El aprendizaje por proyectos y el trabajo colaborativo, como herramientas de aprendizaje, en la construcción del proceso educativo, de la Unidad de aprendizaje TIC'S. Revista Iberoamericana para la Investigación y el Desarrollo Educativo: RIDE, 3(5), 123-138. Recuperado de: https://dialnet.unirioja.es/servlet/articulo? codigo $=4932698$

López, V.M. (2012). Evaluación formativa y compartida en la universidad: clarifi- cación de conceptos y propuestas de intervención desde la Red Interuniversitaria de Evaluación Formativa. Psychology, Society, \& Education, 4(1), 117-130

Aprendizaje basado en proyectos y su evaluación formativa en la asignatura Fundamentos y Estrategias

Didácticas de la Educación Musical. Una buena práctica en la Universidad de Valladolid 
Martí, J.A., Heydrich, M., Rojas, M., \& Hernández, A. (2010). Aprendizaje basado en proyectos: una experiencia de innovación docente. Revista Universidad EAFIT, 46(158), 11-21. Recuperado de:

http://www.redalyc.org/pdf/215/21520993002.pdf

Monreal, I. M., \& Sobrino, D. (2014). La canción de autor en las áreas de música y geografía e historia en educación secundaria. Eufonía. Didáctica de la Música. $62,63-71$.

Pérez, M. M. (2008). Aprendizaje basado en proyectos colaborativos. Una experiencia en educación superior. Laurus, 14(28), 158-180.

Pérez, A., Julián, J. A., \& López, V. (2009). Evaluación formativa y compartida en el EEES. En V. López (Ed.), Evaluación compartida en Educación Superior. Propuestas, técnicas, instrumentos y experiencias (pp. 21-43). Madrid: Narcea

Zavala, S. (2007). Aprendizaje basado en proyectos: Sistematización de la enseñanza. Recuperado de: http://www.slideshare.net/sistematizacion/aprendizaje-basadoen-proyectos

\section{Referencia de textos legales}

ECD 65/2015, de 21 de enero, por la que se describen las relaciones entre las competencias, los contenidos y los criterios de evaluación de la educación primaria, la educación secundaria y el bachillerato.

Aprendizaje basado en proyectos y su evaluación formativa en la asignatura Fundamentos y Estrategias

Didácticas de la Educación Musical. Una buena práctica en la Universidad de 